\title{
Classification of severe asthma exacerbations: a proposal
}

\author{
C. Picado
}

Classification of severe asthma exacerbations: a proposal. C. Picado. CERS Journals Ltd 1996.

ABSTRACT: The intensity of asthma exacerbations may vary from mild to severe. Many studies have demonstrated that the speed at which severe asthma exacerbation (SAE) develops varies among patients. In some asthmatics, the exacerbation comes on very quickly, whereas in others there is a progressive deterioration of clinical, functional and blood gas parameters.

Sudden SAE are characterised by their explosive presentation and quick recovery. This evolution contrasts with that of patients with a slow onset SAE, who often need prolonged hospitalization. Absence of secretions suctioned from the airways during mechanical ventilation has been reported in sudden SAE, whereas large amounts of viscid mucus are found in patients with the slow onset SAE. The lungs of patients who died during a sudden onset SAE often show empty airways and a predominant neutrophilic infiltration in the bronchial epithelium, in contrast to the presence of abundant eosinophils in patients who died during a slow onset SAE.

Sudden onset SAE may occur as sporadic cases or in outbreaks. Sporadic cases may result from the ingestion of nonsteroidal anti-inflammatory drugs (NSAID) in patients with intolerance to these products, massive exposure to common allergens and ingestion of foods containing sulphites. Asthma outbreaks have been described in many cities. In contrast to sudden onset SAE, slow onset SAE is characterized by a progressive deterioration, accompanied by an increase in the use of bronchodilators. Lack of appropriate monitoring of function by peak expiratory flow (PEF) recording, failure of patients to recognize worsening symptoms and underusage of inhaled and oral steroid treatment have been repeatedly identified as factors which are likely to be associated with slow onset SAE. The contribution of psychosocial problems, depression, denial of asthma severity and nonadherence with the treatment should not be overlooked in patients with slow onset SAE.

Classification of severe asthma exacerbations into two types (sudden onset and slow onset) could help to reveal the aetiology of the attack and may also be relevant to the management of the patient.

Eur Respir J., 1996, 9, 1775-1778.
Servei de Pneumologia, Hospital Clinic, Villarroel 170, 08036 Barcelona, Spain.

Correspondence: C. Picado

Servei de Pneumologia

Hospital Clinic

Villarroel 170

08036 Barcelona

Spain

Keywords: Severe asthma exacerbation status asthmaticus

acute severe asthma

Received: November 241995

Accepted after revision March 91996
Bronchial asthma is a disease characterized by recurrent episodes of bronchial obstruction. The intensity of asthma exacerbations may vary from mild to severe. Clinical symptoms, physical signs, changes in the ventilatory parameters, arterial blood gases and response to bronchodilator therapy are used to evaluate the severity of asthma exacerbations.

Mild episodes of bronchial obstruction commonly occur in most patients and are characterized by chest tightness, rapidly relieved by a beta-adrenergic agent. During moderate exacerbations, the sensation of breathing difficulty is more intense, measurement of the peak flow rate may show a decrease of $100 \mathrm{~L} \cdot \mathrm{min}^{-1}$ or more, and resolution of the crisis may take hours or days, usually requiring bronchodilator therapy and often a short course of oral glucocorticoid. Mild and moderate exacerbations may occur after exposure to known precipitating agents either immunological or nonimmunological (exercise, cold air, irritants), or may be caused by undetectable factors. Mild and moderate exacerbations, especially if they are frequent, usually occur in patients with inadequate control of their asthma, which in some asthmatics results in persistent bronchial obstruction and accentuated bronchial hyperreactivity.

Less frequently, asthmatic patients may suffer severe asthma exacerbations (SAE), characterized by respiratory distress, tachypnoea, tachycardia $\left(>120\right.$ beats $\left.\cdot \mathrm{min}^{-1}\right)$, increased arterial carbon dioxide tensions and respiratory muscle fatigue. A silent chest, cyanosis, bradycardia and confusion or unconsciousness are characteristic signs and symptoms of a SAE. In the past, before the introduction of glucocorticoids and respiratory support in critical care units, some patients could not recover and died during the exacerbation. Other patients followed a protracted course with a slow resolution of the attack.

\section{Status asthmaticus and severe acute asthma}

Asthmatic patients suffering a SAE were considered to be in status asthmaticus. The term "status asthmaticus" was originally used to describe a SAE which had continued for more than $24 \mathrm{~h}$. Two distinctive features were usually used to identify status asthmaticus: severity 
and lack of response to bronchodilators. Although the latter condition is usually present in patients with a progressive SAE, it is not always present in patients with a sudden onset exacerbation and, thus, the term status asthmaticus basically implies severity. However, bronchial asthma may also be considered severe when bronchodilators, high doses of an inhaled glucocorticoid and oral steroids are needed to relieve persistent symptoms.

The term "severe acute asthma" was introduced into the literature several years ago by British authors, to differentiate severe exacerbations from chronic severe asthma [1]. The term rapidly gained the favour of chest physicians in Europe but not in the USA, where the term status asthmaticus is still in use. To some extent, however, the term severe acute asthma may be considered misleading because many studies have demonstrated that the speed at which a SAE develops varies among patients $[2,3]$.

\section{Sudden onset and slow onset severe asthma exacerbations}

In some asthmatics, exacerbation comes on very quickly, whereas in others there is a progressive deterioration of clinical, functional and blood gas parameters [3-6]. To differentiate between both types of severe asthma attacks other terms have been proposed. "Acute SAE" and "subacute SAE" [2, 4] or "sudden onset" and "slow onset" severe asthma attack [7] are examples of these proposals.

Sudden SAE are characterized by their explosive presentation in otherwise clinically stable patients. There

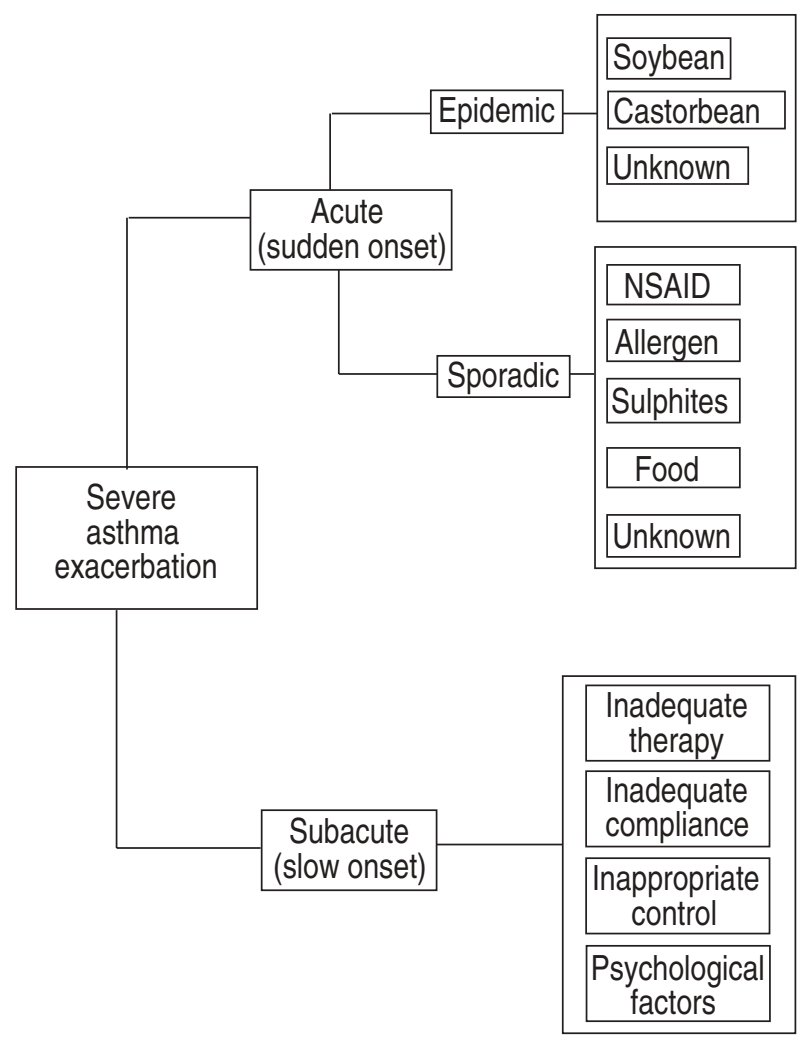

Fig. 1 - Classification and predisposing factors of severe asthma exacerbations. NSAID: nonsteroidal anti-inflammatory drug. appears to be a relationship between the duration of the exacerbation and the period of recovery $[4,5]$. Sudden SAE patients frequently recover very quickly, and even patients requiring assisted ventilation can often be extubated a few hours later $[4,5]$. This evolution contrasts with that of patients with a slow onset SAE, who often need hospitalization for several days before full recovery occurs $[4,5]$. Absence of secretions suctioned from the airways during mechanical ventilation has been reported in sudden SAE in contrast with the observation of large amounts of viscid mucus in patients with the slow onset SAE [5]. Pathological examination of the lungs of patients who have died during a sudden onset SAE often show empty airways and it is assumed that the patients have died of extreme bronchoconstriction. It is interesting to note that a recent study has also found differences in the histological characteristics of sudden and slow onset SAE. In patients dying as a consequence of an acute SAE, a predominant neutrophilic infiltration in bronchial epithelium was detected in contrast to the presence of abundant eosinophils in patients who had died during a slow onset SAE [6]. All these differences support the notion that SAE can be differentiated into two clearly defined entities, acute (sudden onset) and subacute (slow onset) SAE (fig. 1).

\section{Sporadic and epidemic sudden onset severe asthma exacerbations}

Interestingly, sudden onset SAE may occur as sporadic cases or in outbreaks. Sporadic cases may result from the ingestion of a nonsteroidal anti-inflammatory drug (NSAID) in patients with intolerance to these products. In $8 \%$ of patients with near-fatal asthma requiring mechanical ventilation, the exacerbation had been precipitated by these compounds [8]. However, aspirin and related drugs are systematically ignored in papers discussing predisposing factors for developing fatal or near-fatal asthma attacks. A recent paper clearly reveals that aspirin-induced asthma is not explored in acute SAE. MARQuetTE et al. [9] retrospectively evaluated 147 asthmatic patients who underwent mechanical ventilation to treat a life-threatening asthma attack. The authors were surprised to find that aspirin-sensitivity was mentioned in $24 \%$ of the patients' clinical records. However, the most striking finding was that aspirin or related drugs had not been recorded in any of the patients as a precipitating factor of the severe attack. In their discussion, the authors pointed out that "unfortunately, we did not investigate the causative role of aspirin or nonsteroidal anti-inflammatory drug ingestion in the attacks of the patients reported here".

Sudden death both in children and adults with asthma is frequently reported in medical journals and the potential factors responsible for these usually preventable deaths have been debated. Although nonsteroidal drugs may be responsible for some deaths [4], NSAID-induced SAE is never mentioned in these papers, probably because, as the MARquetTE et al. [9] study suggests, the possible role of NSAID as a precipitating factor has not been thoroughly investigated.

Other potential precipitants of sudden onset SAE are hydrocortisone in aspirin-sensitive patients [10], and massive exposure to common allergens [11]. O'Hollaren et 
al. [12] described several patients with severe and sometimes repetitive acute SAE probably precipitated by exposure to spores of Alternaria alternata. Rare episodes of sudden SAE precipitated by certain foods have also been reported [13]. Ingestion of foods containing sulphites may also be implicated in the production of acute SAE [14]. Nevertheless, in many patients suffering sudden onset SAE the precipitating factor cannot be detected.

Epidemic asthma outbreaks have been described in many cities: New Orleans [15], New York [16], Melbourne [17], Birmingham [18], London [19], Barcelona [20], Naples [21], Cartagena (Spain) [22], and Toledo (USA) [23]. Soybean [20-22] and castor bean [23] are wellestablished causes of asthma outbreaks. Airborne fungal spores, especially Didymella exitidis and Sporobolomyces [18], and rye grass pollen grains [17] have also been implicated in some urban episodes of epidemic asthma, but definite proof of their implication in asthma outbreaks is still lacking .

The SAE on epidemic days are characterized by their sudden onset. Some patients suffered SAE requiring admission into the intensive care unit, and frequently the most severe cases underwent immediate intubation and assisted ventilation [24]. However, most patients with severe exacerbations of epidemic asthma had a very rapid recovery and most of the patients who underwent mechanical ventilation could be extubated a few hours later [24].

Epidemic asthma has only been described in large cities. Epidemic episodes affecting a limited number of patients may occur in middle-sized or small towns and remain undetected because a few asthmatic patients with a sudden onset severe asthma attack, requiring attendance in the emergency room in two or more hospitals will rarely, if ever, be considered as an unusual phenomenon by physicians on call [25].

The similarities between epidemic asthma and sporadic sudden onset SAE precipitated by massive exposure to allergens suggest that atmospheric allergens might be responsible for many attacks usually considered idiopathic [25]. Recent studies showing that atopic asthmatics are more predisposed than nonatopic to develop rapid and fatal asthma attacks also suggest that perhaps the presence of allergens in the environment surrounding the patient may be the precipitating factor of some asphixic attacks $[26,27]$. Unfortunately, and in contrast to indoor allergens, it is very difficult, if not impossible, for physicians and patients to establish a cause-effect relationship when the precipitating agent is an outdoor allergen.

There are many articles reporting on patients who suffer recurrent sudden SAE. In most of these papers, more interest is devoted to clinical and therapeutic aspects than to the investigation of the aetiology of the repetitive episodes. There is a considerable body of experience from epidemic and nonepidemic asthma precipitated by allergen exposure suggesting that environmental allergens may be the agents responsible for some of these episodes. Thus, when dealing with one of these patients, the possible contribution of undetected allergens must not be overlooked, and patients should be thoroughly investigated in order to discover the aetiological agent. If two or more asthmatic patients are repeatedly attending the emergency room on the same day, the possibility that an environmental allergen is causing the asthma attack should be suspected [25]. Many reports have described patients who suffer recurrent severe asthma attacks without apparently responding to treatment. Perhaps the erratic oscillation of symptoms and peak expiratory flow (PEF) is a reflection of changes in the concentration of environmental agents. Interestingly enough, a recent paper reported that patients with severe brittle asthma are highly atopic when measured by either skin-prick test or allergen specific immunoglobulin E (IgE) [28]. The predominant role of allergen exposure in sudden onset SAE is reinforced by recent studies which report a strong correlation between acute asthma attacks and high levels of indoor antigens, such as cats, dust mites, and cockroaches [29].

\section{Slow onset severe exacerbation of asthma}

In contrast to sudden onset SAE, slow onset SAE is characterized by a progressive deterioration accompanied by an increase in the use of bronchodilators [4-6]. The initial history of many patients may suggest that they had suffered a sudden deterioration, but a more detailed history of the evolution of the attack reveals increasingly severe symptoms over several days, or even weeks. Most, if not all, of these patients benefit from a short course of steroids. Lack of appropriate control of symptoms by PEF monitoring, failure of patients to recognize worsening symptoms and underusage of inhaled and oral steroid treatment have been repeatedly identified as factors which are likely to be associated with slow onset SAE. The contribution of psychosocial problems, depression, denial of asthma severity and nonadherence with the treatment should not be overlooked in patients with slow onset SAE [30-33].

\section{Potential interests of a new classification for severe asthma exacerbation}

Concern about asthma death is increasing. Most of the deaths are avoidable if patients are adequately investigated and treated [7]. However, prevention of attacks and adequate treatment require previous understanding of the mechanisms by which severe asthma exacerbations develop, and the identification of risk factors in these attacks. Classification of SAE into two types (acute or sudden onset, and subacute or slow onset) could help to reveal the aetiology of the attack and may also be relevant to the management of the patient (fig. 1). In patients with sudden onset SAE, NSAID sensitivity, ingestion of sulphites and allergen exposure should be thoroughly investigated. In patients with repetitive sudden SAE, environmental precipitating allergens should be suspected, especially when these "repeaters" coincide on one or more occasions with other patients in the emergency room. In patients with slow onset SAE, faults in management should be investigated and corrected. These patients will benefit from written detailed plans, home PEF monitoring and therapy in accordance with the most recent guidelines for asthma treatment.

\section{References}

1. Grant I. Severe acute asthma or acute severe asthma. BMJ 1983; 287: 873. 
2. Picado C. Severe acute asthma or acute severe asthma. BMJ 1983: 287: 874

3. Arnold AG, Lane DJ, Zapata E. The speed of onset and severity of acute severe asthma. Br J Dis Chest 1982; 76: $157-163$.

4. Picado C, Montserrat JM, Roca J, et al. Mechanical ventilation in severe exacerbation of asthma: study of 26 cases with six deaths. Eur J Respir Dis 1983; 64: 102-107.

5. Wasserfallen JB, Schaller MD, Feihl F, Perret CH. Sudden asphyxic asthma: a distinct entity? Am Rev Respir Dis 1990; 142: 108-111.

6. Sur S, Crotty TB, Kephart GM, et al. Sudden-onset fatal asthma: a distinct entity with few eosinophils and relatively more neutrophils in airway submucosa. Am Rev Respir Dis 1993; 148: 713-719.

7. Strunk RC. Death due to asthma: new insights into sudden unexpected deaths, but the focus remains on prevention. Am Rev Respir Dis 1993; 148: 550-552.

8. Picado C, Castillo JA, Montserrat JM, Agusti-Vidal A Aspirin-intolerance as a precipitating factor of life-threatening attacks of asthma requiring mechanical ventilation. Eur Respir J 1989; 2: 127-129.

9. Marquette $\mathrm{CH}$, Saulnier F, Leroy O, et al. Long-term prognosis of near-fatal asthma. Am Rev Respir Dis 1992; 146: 76-81.

10. Partridge MR, Gibson GJ. Adverse bronchial reactions to intravenous hydrocortisone in two aspirin-sensitive asthmatic patients. $\mathrm{Br}$ Med J 1978; 1: 1521-1522

11. Spinozzi F, Grignani F. Exposure to an aeroallergen and respiratory arrest in patients with asthma. $N$ Engl J Med 1991; 325: 306-307.

12. O'Hollaren MT, Yunginger JW, Offord KP, et al. Exposure to an aeroallergen as a possible precipitating factor in respiratory arrest in young patients with asthma. $N$ Engl J Med 1991; 324: 359-363.

13. Sampson HA, Mendelson L, Rosen JP. Fatal and nearfatal anaphylactic reactions to food in children and adolescents. N Engl J Med 1992; 327: 380-384.

14. Bush RK, Taylor ST, Busse W. A critical evaluation of clinical trials in reactions to sulfites. J Allergy Clin Immunol 1985; 78 (Part 2): 191-202.

15. Salvaggio JE, Klein RC. New Orleans asthma. I. Characterization of individuals involved in epidemics. $J$ Allergy 1967; 39: 227-234.

16. Goldstein IF, Cuzick J. Daily pattern of asthma in New York and in New Orleans: an epidemiological investigation. Environ Res 1983; 30: 211-223.

17. Morrison I. It happened one night. Med J Aust 1960; 1: $850-851$
18. Packe GE, Ayres JG. Asthma outbreak during a thunderstorm. Lancet 1985; ii: 199-204.

19. Murray V, Venables K, Laing-Morton T, Partridge M, Williams D. Epidemic of asthma possibly related to thunderstorms. Br Med J 1994; 309: 131-132.

20. Antó JM, Sunyer J, Rodriguez-Roisin R, Suarez-Cervera M, Vazquez L. Community outbreaks of asthma associated with inhalation of soybean dust. N Engl J Med 1989; 320: 1097-1102.

21. D'Amato G, Liccardi G, Cazzola M. Environment and development of respiratory allergy. Monaldi Arch Chest Dis 1994; 50: 406-411.

22. Navarro C, Marquez M, Hernando L, Galván F, Zapatero L, Caravaca F. Epidemic asthma in Cartagena, Spain, and its association with soybean sensitivity. Epidemiology 1993; 4: 76-79.

23. Figley KD, Elrod RH. Endemic asthma due to castor bean dust. JAMA 1928; 90: 79-82.

24. Picado C. Barcelona's asthma epidemics: clinical aspects and intriguing findings. Thorax 1992; 47: 197-200.

25. Picado C. Barcelona's asthma epidemics. Thorax 1992; 47: 843-844.

26. British Thoracic Society. Comparison of atopic and nonatopic patients dying of asthma. Br J Dis Chest 1987; 81: 30-34.

27. Jenkins PF, Mullins J, Davies BH, Williams DA. The possible role of aeroallergens in the epidemic of asthma deaths. Clin Allergy 1980; 11: 611-620.

28. Miles JF, Cayton RM, Ayres JG. Atopic status in patients with brittle and nonbrittle asthma: a case control study (Abstract). Am Rev Respir Dis 1994: 149: A245.

29. Pollart SM, Chapman MD, Fiocco GP, Rose G, PlattsMills TA. Epidemiology of acute asthma: IgE antibodies to common inhalant allergens as a risk factor for emergency room visits. J Allergy Clin Immunol 1989; 83: 875-882.

30. Miller BC. Depression and asthma: a potentially lethal mixture. J Allergy Clin Immunol 1987; 80 (part 2): 481-486.

31. Rea HR, Scragg R, Jackson R, Beaglehole R, Fenwick J, Sutherland DC. A case-control study of deaths from asthma. Thorax 1986; 41: 833-839.

32. Picado C, Montserrat JM, de Pablo J, Plaza V, AgustiVidal A. Predisposing factors to death after recovery from a life-threatening asthmatic attack. J Asthma 1989; 24: 231-236.

33. Irwin RS, Curley FJ, French CL. Difficult-to-control asthma: contributing factors and outcome of a systematic management protocol. Chest 1993; 103: 1662-1669. 